\title{
A REVIEW ON VIBRATION ANALYSIS OF ROTOR SHAFT SYSTEM BY VARIOUS FAULT DIAGNOSIS TECHNIQUES
}

\author{
BEHARA SANTOSH SAGAR
}

Assistant Professor, Department of Mechanical Engineering, GMRIT, Andhra Pradesh, India

\begin{abstract}
Rotating machinery is commonly used in different kinds of mechanical applications. As a promising field for the unwavering quality of the current mechanical frameworks, strategies for early fault diagnosis (EFD) have attracted consideration from both the scholarly community and industry. EFD is essential to provide adequate data to undertake essential support activities, thereby preventing serious disappointments and reducing losses related to money. In the last two decades a gigantic measure of the examination work has been led to the creation of EFD methods. This paper reviews and summarizes the exploration taking a shot of apparatuses, rotors and course at EFD. The main motivation behind this paper is to fill out early-deficiency analysis as a guide for scientists.
\end{abstract}

KEYWORDS: Early fault diagnosis; Signal processing; Rotating machinery; Feature extraction.

Received: Jun 08, 2020; Accepted: Jun 28, 2020; Published: Sep 16, 2020; Paper Id.: IJMPERDJUN20201281

\section{INTRODUCTION}

For example, blowers, steam turbines, vehicles, current fans and aircraft engines, regular resident and military applications, rotating machinery is the most commonly used type of machine in the present business. Because of the high help load, the cruel working conditions or the inevitable weariness, the pivoting hardware can create weaknesses.On the unlikely chance that the error cannot be evaluated easily, it may cause the entire system to be shut down and even puzzlingly catastrophic. In this way, it is noteworthy to identify blames early and to evaluate the degree of deficiency as ahead of schedule as feasible to avoid cataclysmic malfunctions and to guarantee the safe operation of the system.

Numerous scientists are focused on increasing methods of early fault diagnosis(EFD) to screen pivoting hardware well-being states.As of now, various EFD procedures have been successfully applied in cutting-edge organizations, such as the vibration-based EFD method, current EFD methodology, acoustic release-based EFD procedure, sound-based EFD system, torque-based EFD technique, and encoder-dependent turning of EFD technique etc.Among these sensor signals, vibration-based definite strategy is the most generally concentrated since vibration signs can legitimately speak to the dynamic conduct of the rotating device. Nonetheless, the early lack of signal is consistently too low as a result of the small amount of mischief that can cause minimal periodic main thrusts.

In order to distinguish the condition of well-being of the machine, the discovery of deficiencies and the disconnection of deficiencies are constantly used. More forensic research on Fault Detection and Diagnosis (FDD) has been performed as of late. The explanation for FDD is explained by on-line observation of steam turbine working states.In this way, the accuracy of estimating gadgets / instruments is significant and it is fortunate that 
both OEMs and plant owners give due weight to determine these instruments. Additionally, progressions in PCs and microchips help bring about an effective programmed detection and diagnosis of faults.

The methodology used in FDD 's conduct relies on using utilitarian rather than physical excess. This includes the implicit excess between the system inputs found in the links, and quantifiable yields are used for FDD. Whether the translation for different FDD-related exercises is clearly determined,the errands of shortcoming recognition and deficiency determination are interconnected to one another. A fault can be characterized in terms of its seriousness and event into a sudden deficiency and a nascent issue. Sudden issue alludes to the complete loss of detecting signs or unrefined changes in elements of various kinds. The emerging deficiency alludes to slow changes in the framework's process boundaries, prompting expected breakdowns. The event of the generally small extent and moderate time shifting properties makes it harder to identify starting deficiencies than unforeseen faults. The FDD is composed of old style and propelled procedure. The three capacities that accompany it can be recognized as old style techniques: checking, programming security and managing with determination of shortcomings. If observation should occur, estimated factors are checked for setting focuses, and alerts are generated for the administrator. Programmed assurance begins an appropriate neutralization if a perilous state of the procedure should occur.Finally, fault analyzed management performs balancing choices depending on estimated factors, determined highlights, created side effects.

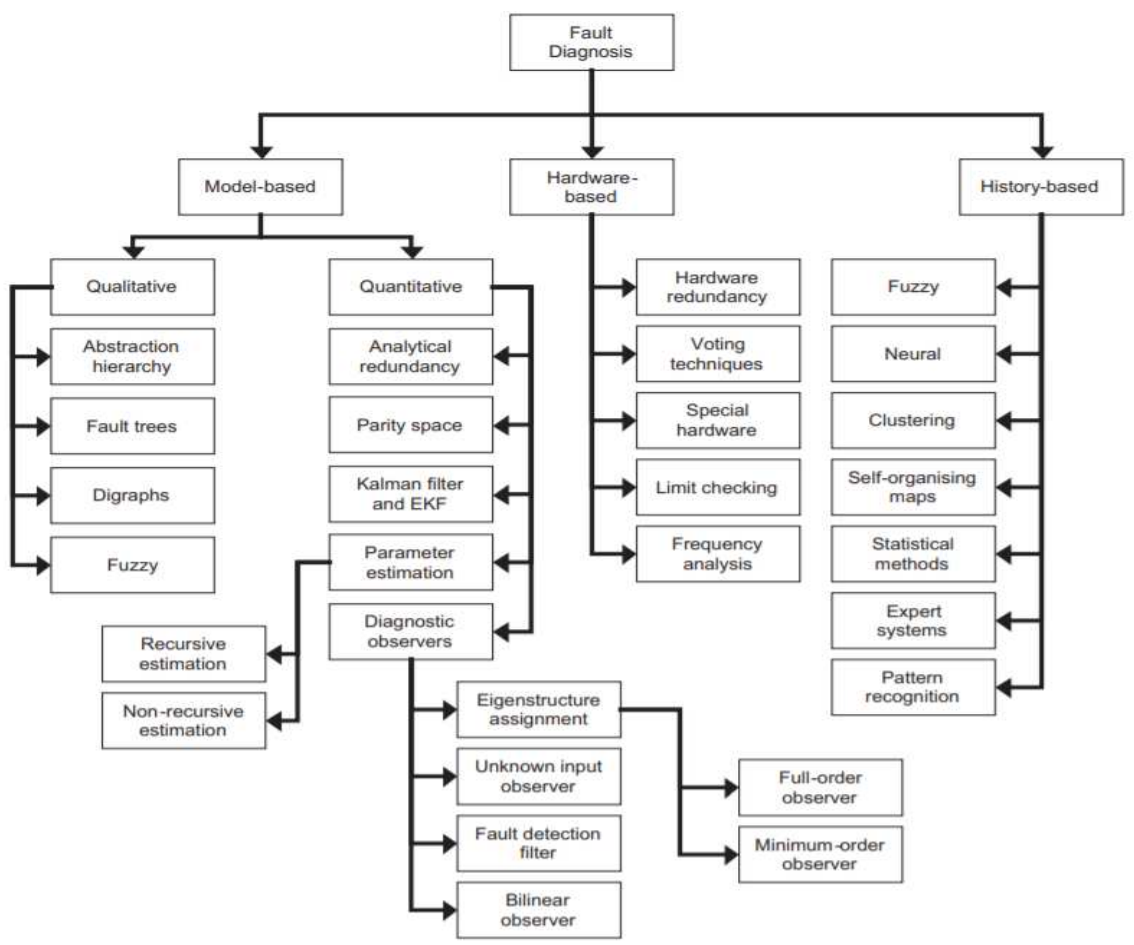

Figure 1: Classification of Fault Diagnosis Methods.

A top-to-bottom fault analysis is typically unrealistic, despite the fact that these old style set focuses based FDD techniques are basic and solid, they can just be ready to respond after a generally huge difference in an element, and further.Along these lines, propelled FDD techniques needed with unexpected or nascent conduct of time for early location of small damages. Present day-to-day oversight ideas allow for checking and programming security, but can also help with performing on-demand support or a previous step-by - step recognition of creating faults. They can be utilized in shut circle circumstances and work considerably under unique activity. 
Testing the pivoting hardware vibration characteristics assists in detecting issues that could be producing. One of the most significant machines for producing power is the Steam Turbo-Generator. The constant problem analysis is becoming increasingly important for steam turbine generators and various turning devices such as generators, engines, siphons, cooling fans, etc. Vibration analysis is one of the widely used and viable techniques for fault identification and conclusion of various pivoting apparatuses. Vibration research is helpful in the diagnosis of unbalance, bowing and misalignment in shaft and rotor, damage and loss of bearing fat, tooth failure, worn teeth, equipment misalignment and mechanical detachment.

There is an extraordinary amount of composing on powerful fault diagnosis frameworks that extends from logical strategies to artificial intelligence and truthful approaches. There are techniques that require precise frameworks (plants), quantitative models or subjective models from a planned displaying. Notwithstanding, there are techniques that don't require any type of model data and depend just on memorable framework data.

\subsection{Model-Based Fault Diagnosis}

Model-based fault diagnosis techniquemethods typically move on a model made based on some exceptional understanding of the material science of the plant or the strategy. If in doubt, systems for model-based fault ending are emotionally or quantitatively appointed to a large extent.

\section{Qualitative Methods}

Qualitative model-based fault diagnosis techniques use a model where the relationship between data yields of the plant is expressed to the degree that emotional limits circulate around various units all the while. In complex consultation system, problem trees, digraphs, and fuzzy structures, Qualitative model-based fault diagnosis is thoroughly presented.

\section{Quantitative Methods}

Quantitative model-based fault diagnosis techniques use a model where the relationship between the data yield and the plant is undoubtedly conveyed.

\subsection{Hardware-Based Fault Diagnosis}

Hardware-based machinery diagnosis techniques don't give a numerical model of plant or process material science.

\subsection{History-Based Fault Diagnosis}

History-based techniques of fault diagnosis do not send a numerical model of plant or procedure material science, yet a model has obtained information from the known and estimated info and yield process.

The finding of deficiencies is an important subject for and by research. Current systems have an extraordinary weight to keep reducing unplanned personal time, abuse of execution, and perils of well-being that involve separating and recovering from anticipated blames as early as possible.We break down and summarize ongoing advances in fault analysis, order them in subtleties and even more thoroughly, and discuss the current issues and new difficulties of finding faces in the huge information age. In this paper, we first extensively review and study the improvement of the diagnosis of fault.It would be necessary to meld the advantages of various demonstrative techniques and make more use of the separate problem prospects, for example, forming an indicative control system for a shut-circle and ultimately deciding on upkeep choices. This makes future inquiries contemplative.

\section{LITERATURE REVIEW}


The rotating machinery EFD has drawn considerable consideration from both the academic and industrial worlds. As indicated by the distinguishing of the execution route for early pivoting apparatus fault analysis, we can divide it into two primary classifications: FFD techniques and AI strategies. We will briefly introduce the Fault diagnosis approaches and their applications in rotating machinery,influence of vibration analysis in condition monitoring and fault diagnosis of rotating shaft,Bend shaft fault diagnosis, Shaft misalignment fault diagnosis,Static Unbalance fault diagnosis,Diagnosis of shaft cracks and cavitations, Rotor rub fault diagnosis and different ways of vibration measurement like the usage of electromagnetic exciters, time frequency analysis, wavelet techniques, on-shaft vibration measurement.

\subsection{Fault Diagnosis Approaches and their Applications in Rotating Machinery}

[1] provides a framework and approach that has been evaluated on a research scheme. As suggested by the relative feedback, it was discovered that the Classifier Chain method is the most appropriate for synchronous noisy misalignment and fault unbalance issues. The fault marks are made of deficiency extent and upset commotion frequencies and some extra boundaries.

[2] check the transferability of the said procedures to a wider variety of rotor-related blames on specific machines. Additionally, a new Multi-Speed Multi-Foundation (MSMF) technique is proposed which encourages FD to immediately analyze vibration information from equally built machines with various powerful qualities operating at different consistent state speeds. It is seen that the strategies proposed recently are surely ready to isolate the scope of conditions tried on machines with different unique attributes.

[3] offers a detailed overview of the best in class of techniques for deficiency study, regarding turning machines. Fault finding is an extremely wide-ranging topic that allows for the complete inclusion of the vast number of regions associated with this area.

In [4] the bearing issues in pivoting hardware are tentatively investigated using specific time, frequency, and timefrequency vibration investigation procedures. With respect to Fast Fourier FFT and Inverse Simple Fourier shift IFFT, the data signal acquired from the turning hardware with moving part path which is metal roller with internal and external track deviations in examination with wellbeing direction is broken down.

In [5] several turning apparatus EFD procedures are studied as far as the early fault diagnosis based on FFD and early fault diagnosis based on AI are concerned. At last, some new possibilities for examination are being called to attention. It is agreed that this audit mixed the state of craftsmanship references on EFD to transform hardware for per users inspired by this region of exploration. Meanwhile pivoting work on EFD with the development of sign planning techniques.

\subsection{Influence of vibration analysis in condition monitoring and fault diagnosis of rotating shaft}

In [6] as a model for a basic turning machine, a test rig was worked up. The technique developed for estimating on-shaft vibration has been widely explored. Because of the sensor's rotational motion, it was found to have equilibrium, a prevailing gravity impact despite a high degree of interaction between the ghastly songs.

[7] provides point-by - point data on the effect of various misalignments on rotor vibration and stator current response. Orbit vibration and FFT plots of both vibration as well as current are used to recognize special highlights identified with deficiency of misalignment. Vibration algorithm and current reaction found the enormous impact on the 
symphonic content of the system that was brought on by the different kinds of misalignments.

[8] mainly handles the complex investigation of the rotor shaft structure which is functionally graded (FG). Force law degree is received for demonstration of FG rotor shaft numerical material. Timoshenko beam theory (TMBT) is also used for the display of the FG shaft with a functionally evaluated (FE). The layout of the FG shaft was tested by comparing simple levels with the usable writing.

[9] proposes a functioning vibration control conspire to control a rotor shaft's transverse vibration due to unbalance and presents a hypothetical report. The procedure uses electromagnetic exciters mounted on the stator of an aircraft, when everything is said in the ordinary areas of assistance, around the rotor shaft for applying appropriate power of activation over an air hole to control transverse vibration.

[10] discussed the various procedures used to conclude shortcomings depending on the vibration investigation method. Time space strategies do not provide any indicative evidence under fluctuating responsibility conditions. The groups of weakness are hard to identify by FFT strategy. Analysis of the programmed issues can be performed using ANN and Support Vector Machine. It also gives better order of the different problems.

\subsection{Bend Shaft Fault Diagnosis}

[11] proposed vibration investigation method used for bowed shaft diagnosis. Both step and sufficiency are obtained in application analysis. The type and area of defects are usually recognized from stage and abundance. Exploratory results show request exam is a powerful bowed shaft procedure.

\section{- Shaft Misalignment Fault Diagnosis}

A conceptual model rational to determine the vibratory reaction of smart shaft rotors associated with an adaptable coupling has been developed in [12] Considering the ghastly inspection is a basic method for examining the mechanical state of the apparatus, the normal vibration spectra for two adaptable couplings, a three-pin Reynold and a three-jaw Lovejoy coupling, were broke down.

\section{- Static Unbalance Fault Diagnosis}

In [13] a model-based fault-finding technique is created to discern the problem couch rotor - coupling - bearing frame subject to misalignment and unbalance at consistent state condition. This model-based fault-finding method depends on the extravagantly depicted lingering period.

\section{- Diagnosis of Shaft Cracks and Cavitation's}

A problem analysis technique based on multi-input convolutional neural network (MI-CNN) is proposed in [14] focusing on the problem of recognizing the pole misalignment and breaking in a rotor system.

\section{- Rotor Rub Fault Diagnosis}

[15] Tests consistent state vibration reaction of the rotor bearing frame with rotor blames, e.g., unbalance, break, rotorstator friction, and misalignment at sub-basic rotational speed. It is for this purpose that test rigs are designed and developed. The customary Fourier range (i.e., FFT) has constraints in showing the spin nature (i.e., forward/in reverse spin) of the rotor deficiencies.

\subsection{Signal Processing Methods used in Vibration-Based Condition Monitoring}


[16] proposes the development of a GE MS 3002 gas turbine-type fault monitoring device based on vibration analysis techniques using spectral analysis instruments. The outcomes got show the adequacy of the checking device approach introduced on the gas turbine to keep away from vibration mode activity and to produce ideal execution during gas turbine activity.

In [17] the technique for relating pivoting machine surrenders with the vibrations gathered and showed by the various kinds of analyzers is given consideration utilizing vibration investigation, the state of a machine can be continually monitored. Detailed breaks down might be led to survey a machine's security and order any shortcomings that may happen or as of now exist. [18] includes Process Power Spectrum Entropy (PPSE) and Support Vector Machine (SVM) (PPSESVM) for the analysis of rotor vibration disappointment and ongoing vibration monitoring. Based on the PPSE procedure, the PPSE esteems (for example, data highlights) of this information are resolved to make singular vectors as tests for issue determination.

[19] provides an outline of vibration examination and deficiency finding in different turning machine segments just as endeavors to encapsulate ongoing work and headways in pivoting gadget vibration investigation methods. Frequency area methods are equipped for distinguishing rotor issue areas.

[20] proposes the utilization of time-frequency (TF) changes for breaking down vibration information in bearingcurrent engines. The exploratory outcomes acquired in genuine machines show that vibration examination through T-F instruments can give critical preferences in distinguishing current harm to orientation; among other things, these methods permit the slow debasement of a heading to be imagined while giving viable separation versus other non-issue related segments.

Two kinds of dynamic models for shaft misalignment of pivoting hardware for vibration examination and shaft misalignment state analysis are proposed in [21]. The strategy for algorithm of vibration powers brought about by misalignments is additionally appeared to get the arrangement of the dynamic models and to explain the vibration signal qualities estimated in the pole misalignment state.

\subsection{Different Ways of Vibration Measurement Like the Usage of Electromagnetic Exciters, Time Frequency} Analysis, Wavelet Techniques and on-Shaft Vibration Measurement

[22] detect various deficiencies in the rotor system using run-up vibrations. In this study, both re-enactments and tests are misaligned, rotor-stator rub problem recognition is considered. The use of HHT algorithm is featured in conjunction with the STFT and CWT approaches singing the relative report. It is examined the effect of sign to noise ratio to identify vulnerabilities using the three-time frequency procedures. The shortcomings of defects deemed exceptional for leisure research and trial research.

[23] presents the Empirical mode decomposition (EMD), time waveform and power spectrum density (PSD) investigation for the identification of limited prod gear faults. At first, the test model was produced at different RPMs for vibration investigation of single tooth breakage of spike gear and subsequently explicit question was presented in driven rigging under various harm conditions.

[24] stress the significance of correct frameworks for vibration estimations. In general, the prerequisites provided by the ISO 10817-1 specification should be retained. The most important requirements are the weakness thresholds which should not exceed \pm 10 percent and the resistance ranges of the channel frequency which should not exceed $\pm 3 \mathrm{db}$. It is 
conceivable to apply relative shaft vibration estimations, however it is disagreeable. Vibration estimations can be executed with on the web or disconnected estimation frameworks.

In [25] vibration work is commonly used in prescient support programs including siphons, generators, gearboxes, motors, fans, and blowers, as well as a wide variety of cars, daunting machines, spans, and structural building structures. Excessive vibration of under-burden hardware can be caused by wear, consumption, or even natural powers, and is a significant reason for disappointment with hardware or structures.

[26] review vibration testing strategies and investigate their capabilities, favorable circumstances and inconvenience in the control of moving component bearings. A Comparative Study of Temporary Analysis, FFT, CA, EA and WT for the determination of bearing issues has been introduced to investigate the vibration investigation procedures and their capabilities; preferences, and hindrance in observing moving component orientation.

[27] investigate the link between the investigation of vibration and the finding of deficiencies. This was accomplished by examining the vibrations and exploring an exploratory electro-motor'sdistinctive working states. As a similar test, the electromotor was initially run under ordinary working conditions. A progression of tests compared with different working conditions was then led.

\section{MAJOR FINDINGS AND CONCLUSIONS}

The paper focused on those techniques that are settled in the community of building and exploration as a result of the gigantic movement of ebb and flow exams in this field. In particular, strategies for the determination of faults were grouped into three fundamental classes: model-based, hardware-based, and historical methods.Model-based deficiency finding techniques have the accompanying qualities: They have the most reliable estimators or observers when updated all over, point-by - point models can show realistic normal and faulty errors, and casing grips can appear, and errors can be displayed and fused into ineffective investigation procedures.They do have the accompanying deficiencies: experimental models could be perplexing and thus computationally concentrated; showing losses could have a major effect on the final results. The accompanying virtues are the finding techniques for hardware-based issues: quick and modest to implement, very perceived and trusting. They also have the shortcoming of Additional hardware.

High costs of maintenance and extra room required to require additional equipment. The accompanying qualities are historically based fault diagnostic techniques: suitable for fundamentally non-direct systems and do not require knowledge of the material science being demonstrated in the system. They also have the following faults: Models cannot be used after the planning information has been conducted, models are specifically seen in the system and information planning is required from both ordinary and broken operations. This is basically an issue of the nature of the accessible scientific model of the system,knowledge of the framework and framework structure. What's more, the attainable nature of the separation of issues depends conclusively on the quantity of estimates available.

\section{REFERENCES}

1. Dineva, A., Mosavi, A., Gyimesi, M., \&Vajda, I. (2019). Multi-label Classification for Fault Diagnosis of Rotating Electrical Machines. arXiv preprint arXiv:1908.01078.

2. Nembhard, A. D., Sinha, J. K., \&Yunusa-Kaltungo, A. (2015).Development of a generic rotating machinery fault diagnosis approach insensitive to machine speed and support type. Journal of Sound and Vibration, 337, 321-341.

3. Suresh. V, “FORTIFICATION AND CULPABILITY ANALYSIS OF THREE PHASE INDUCTION MOTOR USING LABVIEW 
“, International Journal of Electrical and Electronics Engineering (IJEEE), Vol . 3, Issue 3, pp. 21-28

4. Edwards, S., Lees, A. W., \&Friswell, M. I. (1998). Fault diagnosis of rotating machinery. Shock and Vibration Digest, 30(1), 4-13.

5. Khadersab, A., \&Shivakumar, S. (2018). Vibration analysis techniques for rotating machinery and its effect on bearing faults. Procedia Manufacturing, 20, 247-252.

6. Wei, Y., Li, Y., Xu, M., \& Huang, W. (2019).A review of early fault diagnosis approaches and their applications in rotating machinery. Entropy, 21(4), 409.

7. Elnady, M. E., Sinha, J. K., \&Oyadiji, S. O. (2012). Faults diagnosis using on-shaft vibration measurement in rotating machines. In The International Conference on Applied Mechanics and Mechanical Engineering (Vol. 15, No. 15th International Conference on Applied Mechanics and Mechanical Engineering., pp. 1-19).

8. Verma, A. K., Sarangi, S., \&Kolekar, M. H. (2013).Misalignment fault detection in induction motor using rotor shaft vibration and stator current signature analysis. International Journal of Mechatronics and Manufacturing Systems, 6(5-6), 422-436.

9. Rao, D. K., \& Roy, T. (2016). Vibration analysis of functionally graded rotating shaft system. Procedia Engineering, 144, 775780.

10. Das, A. S., Nighil, M. C., Dutt, J. K., \&Irretier, H. (2008).Vibration control and stability analysis of rotor-shaft system with electromagnetic exciters. Mechanism and Machine Theory, 43(10), 1295-1316.

11. Sujesh, S., Marulaiah, L., Kumar, K. And Srinivas, K, R. (2018). Vibration based Fault Diagnosis Techniques for Rotating Mechanical Components: Review Paper. IOP Conference Series: Materials Science and Engineering. 376. 012109. 10.1088/1757-899X/376/1/012109.

12. Mogal, S. P., \&Lalwani, D. I. (2017). Fault diagnosis of bent shaft in rotor bearing system. Journal of Mechanical Science and Technology, 31(1), 1-4.

13. Saavedra, P. N., \& Ramirez, D. E. (2004).Vibration analysis of rotors for the identification of shaft misalignment Part 1: theoretical analysis. Proceedings of the Institution of Mechanical Engineers, Part C: Journal of Mechanical Engineering Science, 218(9), 971-985.

14. Jalan, A. K., \&Mohanty, A. R. (2009). Model based fault diagnosis of a rotor-bearing system for misalignment and unbalance under steady-state condition. Journal of sound and vibration, 327(3-5), 604-622.

15. Zhao, W., Hua, C., Wang, D., \& Dong, D. (2020). Fault Diagnosis of Shaft Misalignment and Crack in Rotor System Based on MI-CNN. In Proceedings of the 13th International Conference on Damage Assessment of Structures (pp. 529-540). Springer, Singapore.

16. Patel, T. H., \&Darpe, A. K. (2011). Application of full spectrum analysis for rotor fault diagnosis. In IUTAM symposium on emerging trends in rotor dynamics (pp. 535-545). Springer, Dordrecht.

17. [16] Djaidir, B., Hafaifa, A., \&Kouzou, A. (2017). Faults detection in gas turbine rotor using vibration analysis under varying conditions. Journal of Theoretical and Applied Mechanics, 55(2), 393-406.

18. Girdhar, P., \&Scheffer, C. (2004). Machinery fault diagnosis using vibration analysis. Practical Machinery Vibration Analysis and Predictive Maintenance, 89-133.

19. Anna Szoka, Grzegorz Gajowiec, Waldemar Serbinski \& Andrzej Zielinski, "Effect of Surface State and Stress on an Oxidation of the Zircaloy-2 Alloy “, BEST: International Journal of Management, Information Technology and Engineering (BEST: IJMITE), Vol. 4, Issue 2, pp. 55-64 
20. Fei, C. W., Bai, G. C., Tang, W. Z., \& Ma, S. (2014). Quantitative diagnosis of rotor vibration fault using process power spectrum entropy and support vector machine method. Shock and Vibration.

21. Tenali, N., Babu, P. R., \& Kumar, K. C. K. (2017). Vibrational Analysis in Condition Monitoring and faults Diagnosis of Rotating Shaft-Over View. International Journal of Advanced Engineering Research and Science, 4(1).

22. Prudhom, A., Antonino-Daviu, J., Razik, H., \&Climente-Alarcon, V. (2017). Time-frequency vibration analysis for the detection of motor damages caused by bearing currents. Mechanical Systems and Signal Processing, 84, 747-762.

23. Guan, Z., Chen, P., Zhang, X., Zhou, X., \& Li, K. (2017). Vibration Analysis of Shaft Misalignment and Diagnosis Method of Structure Faults for Rotating Machinery. International Journal of Performability Engineering, 13(4).

24. Chandra, N. H., \&Sekhar, A. S. (2016). Fault detection in rotor bearing systems using time frequency techniques. Mechanical Systems and Signal Processing, 72, 105-133.

25. Akram, M. A., Khushnood, S., Tariq, S. L., Ali, H. M., \&Nizam, L. A. (2019). Vibration Based Gear Fault Diagnosis under Empirical Mode Decomposition and Power Spectrum Density Analysis. Advances in Science and Technology Research Journal, 13, 3.

26. Barszcz, T. (2019). Standard Vibration Analysis Methods. In Vibration-Based Condition Monitoring of Wind Turbines (pp. $33-$ 86). Springer, Cham.

27. Mix, Paul. (2005). Vibration Analysis Method. 10.1002/0471719145.ch12.

28. Boudiaf, A., Moussaoui, A., Dahane, A., \&Atoui, I. (2016). A comparative study of various methods of bearing faults diagnosis using the case Western Reserve University data. Journal of Failure Analysis and Prevention, 16(2), 271-284.

29. Karthik Balasubramanian, Vineeth Chandrasekar, Rahul Menon \& $R$ Sudha, "Intelligent Monitoring of Bushings in anElectrical Transformer “, International Journal of Electrical and Electronics Engineering (IJEEE), Vol. 2, Issue 2, pp.6366

30. Monavar, H. M., Ahmadi, H., Mohtasebi, S. S., \&Hasani, S. (2008). Vibration condition monitoring techniques for fault diagnosis of electromotor with $1.5 \mathrm{~kW}$ power. Journal of Applied Sciences, 8(7), 1268-1273. 

